\title{
Arithmetization of a Circular Arc
}

\author{
Aurélie Richard ${ }^{1}$, Guy Wallet ${ }^{2}$, Laurent Fuchs ${ }^{1}$, Eric Andres ${ }^{1}$, \\ and Gaëlle Largeteau-Skapin ${ }^{1}$ \\ 1 Laboratoire XLIM-SIC, \\ Université de Poitiers, \\ BP 3017986962 Futuroscope Chasseneuil cedex, France \\ \{arichard, Laurent.Fuchs, andres, glargeteau\}@sic.univ-poitiers.fr \\ 2 Laboratoire MIA, \\ Université de La Rochelle, \\ Avenue Michel Crépeau 17042 La Rochelle cedex, France \\ Guy.Wallet@univ-lr.fr
}

\begin{abstract}
In this paper, we present an arithmetization of the Euler's integration scheme based on infinitely large integers coming from the nonstandard analysis theory. Using the differential equation that defines circles allows us to draw two families of discrete arc circles using three parameters, the radius, the global scale and the drawing scale. These parameters determine the properties of the obtained arc circles. We give criteria to assure the 8-connectivity. A global error estimate for the arithmetization of the Euler's integration scheme is also given and a first attempt to define the approximation order of an arithmetized integration scheme is provided.
\end{abstract}

Keywords: Discrete circle, Discrete arc circle, Arithmetization, Numerical scheme, Error order, Connectedness.

\section{Introduction}

The discretization of a curve on a computer screen has been the focus of a lot of research in the late sixties and beginning of the seventies [1, 2]. After that, for a long time, nothing fundamentally new has been proposed as people considered that most of the research in this area had been covered. In 1988, a novel definition of the discrete straight line by J-P. Reveillès [3-5] sparked a new interest in basic discrete geometry and the digitization of discrete curves and surfaces. Many new results especially in the analytical description of discrete objects have been proposed since [ [ $[$ ]. The theoretical roots behind Reveillès' straight line formula can be found in Non Standard Analysis (NSA). This theory [7, 8] provides a framework where infinitely large and infinitely small numbers can be explicitly manipulated. It can be shown that, given an infinitely large number $\omega$ (the global scale), it is possible to establish an equivalence between the set of limited real numbers and a subset $\mathcal{H R}_{\omega}$ of the set $\mathbb{Z}$ of integers. The set $\mathcal{H R}_{\omega}$, with an additional structure, is called the Harthong-Reeb line 99 12]. The intuitive idea behind this concept is to consider $\mathbb{R}$ like $\mathbb{Z}$ seen from far away. 
Many curves can be defined as a solution of the integration of a vector field. With an integration scheme like, for instance, the Euler scheme with an infinitely small integration step, we obtain an infinitely close approximation of the curve. With the help of an arithmetization process consisting in mapping the scheme from the real line to the Harthong-Reeb line, we obtain a discrete curve which is an exact representation of the continuous one. The global scale $\omega$ does not however allow to draw the curve on a computer screen because the points of the curve are infinitely far away from each other. A strongly contracting rescaling in the discrete world allows then a way of getting a scale where the curve can be seen on a computer screen. The approximation that appears in the process of drawing a discrete curve on a screen is not described anymore as a sampling error but as the result of a strongly contracting rescaling in the discrete world. For more details, readers may refer to [13, 14].

At the last DGCI (and in an extended version in the Pattern Recognition journal) [13, 14], we took a new look at the ideas that led to Reveillès' discrete straight line. In this paper we decided to reconsider Holin's work [15 17] on discrete circles generated by the preceding approach. Many different circle generation algorithms exist. Here the purpose is not to propose a new one but rather to understand the continuous-discrete relations.

As a first step, we carefully study the arithmetization of the Euler integration scheme on the differential equation defining circles. The goal of this paper was to apply the insight gained previously on the arithmetization process to a new set of curves. For the discrete straight lines, only one drawing scale appears (labeled $\beta$ where $\omega=\beta^{2}$ ) besides the global scale $\omega$. In the circle case, two different drawing scales appear (labeled $\alpha$ and $\beta$ with $\omega=\alpha \beta$ ). These two drawing scales define two families of discrete circles with different properties.

The connectedness of the discrete circles obtained by such arithmetization methods has never been studied before. In the case of the Euler integration scheme, we show that inside the square $[-\beta, \beta-1]^{2}$ every circular arc of both families ( $\alpha$ and $\beta$ circles) is 8 -connected. This allows a global control over the topological properties of the discrete circle obtained with this method. We also study the quality of the approximation we obtain by the arithmetization of the Euler scheme. We give a way to estimate the error between a circle and its discretization. Thus we define the approximation order of the arithmetized integration scheme that has never been studied before.

This paper is organized as follows: first, we recall the basis about the arithmetization method and we apply it on the circles. Section 3 deals with connectedness conditions while section 4 deals with the error study. A short conclusion and some perspectives are proposed in section 5 .

\section{The Arithmetization Method Applied to Circles}

In this section we recall our arithmetization method [14] using it on the differential equations that describe a circle. We obtain two arithmetized versions of these differential equations that will be the starting point of our study. 
In the Euclidean plane, the circle $C(0, R)$ with center 0 and radius $R \in \mathbb{R}_{+}^{*}$ can be described as the solution of the differential equations:

$$
\left\{\begin{array}{l}
(x(0), y(0))=(0, R) \\
\left(x^{\prime}(t), y^{\prime}(t)\right)=(-y(t), x(t)) .
\end{array}\right.
$$

which express the fact that the tangent vector is orthogonal to the radius vector. These differential equations can be numerically solved using the Euler method. This gives us the following Euler scheme that computes an accurate numerical approximation of $C(0, R)$ when the step $h$ tends to 0 in $\mathbb{R}_{+}$:

$$
\left\{\begin{array}{l}
\left(x_{0}, y_{0}\right)=(0, R) \\
\left(x_{n+1}, y_{n+1}\right)=\left(x_{n}, y_{n}\right)+\left(-y_{n} h, x_{n} h\right) .
\end{array}\right.
$$

The general idea of the arithmetization method [14, 15] is to transform this continuous numerical scheme into a discrete one with only integer variables. This discrete scheme generates a digital planar curve $C_{d}(0, R)$ which is a discrete analog of the circle $C(0, R)$.

This analogy is proved to be theoretically sound using nonstandard analysis [14]. The approach is to perform a rescaling on $\mathbb{Z}$ such that the new unity is an infinitely large integer $\omega \in \mathbb{N}$. The structure we obtain is the HarthongReeb line $\mathcal{H} \mathcal{R}_{\omega}$ [14] which is a numerical system isomorphic to the system $\mathbb{R}_{\text {lim }}$ of limited real numbers (i.e. real numbers that are not infinitely large). The isomorphism is given by the two mappings:

$$
\left\{\begin{aligned}
\varphi_{\omega}: \mathcal{H R}_{\omega} & \longrightarrow \mathbb{R}_{l i m} \\
X & \longmapsto X / \omega
\end{aligned}\right\} \quad \text { and } \quad\left\{\begin{aligned}
\psi_{\omega}: \mathbb{R}_{\text {lim }} & \longrightarrow \mathcal{H} \mathcal{R}_{\omega} \\
x & \longmapsto\lfloor\omega x\rfloor
\end{aligned}\right\}
$$

where $\lfloor x\rfloor$ is the integer part of $x \in \mathbb{R}$ such that $x=\lfloor x\rfloor+\{x\}$ with $0 \leq\{x\}<1$.

Using these mappings, it can be shown that the digital curve $C_{d}(0, R)$ is isomorphic to the initial Euclidean circle $C(0, R)$. However, in this article we are concentrating on the properties of the digital curve $C_{d}(0, R)$ (see [13, 14] for more details on such a proof).

The first step is to describe more precisely the arithmetization of the Euler scheme (2). We suppose that the radius $R$ of the circle is limited $(R \neq+\infty)$ and non infinitesimal $(R \not 0)$. We choose two natural numbers $\omega$ and $\beta$ such that $\omega, \beta$ and $\omega / \beta$ are infinitely large. Let $h=\frac{1}{\beta}(h \simeq 0)$. Using the mapping $\psi_{\omega}$, the Euler scheme is translated to its integer version, the arithmetization of the Euler scheme (2) at the scale $\omega$, by:

$$
\left\{\begin{array}{c}
\left(X_{0}, Y_{0}\right)=(0,\lfloor\omega R\rfloor) \\
\left(X_{n+1}, Y_{n+1}\right)=\left(X_{n}, Y_{n}\right)+\left(-Y_{n} \div \beta, X_{n} \div \beta\right)
\end{array}\right.
$$

where $\div$ is the usual Euclidean division and $X_{i}, Y_{i}, i=1,2, \ldots$ are integer variables. The points $\left(X_{i}, Y_{i}\right)$ define the digital curve $C_{d}(0, R)$.

Unfortunately, the obtained curve $C_{d}(0, R)$ is largely non connected: for instance, if we consider the abscissae $X_{0}$ and $X_{1}$ of the two first points, we have $\left|X_{1}-X_{0}\right|=\lfloor\omega R\rfloor \div \beta$ which is an infinitely large number roughly equal to $\frac{\omega}{\beta} R$. 
This means that the gap between these two points, and in general between two consecutive points of the curve, is infinitely large.

The idea to obtain points that are closer together, is to perform a rescaling from the scale $\omega$ to the intermediate scale $\alpha=\frac{\omega}{\beta}$. To do so, it is convenient to choose $\omega$ as a multiple of $\beta$ : $\omega=\alpha \beta$ with $\alpha \simeq+\infty$. Hence, the rescaling is given by the composition $\psi_{\alpha} \circ \varphi_{\omega}: X \mapsto\left\lfloor\alpha \frac{X}{\omega}\right\rfloor$. This defines two drawing scales $\alpha$ and $\beta$ because one can of course also consider a rescaling to the scale $\beta$.

Since $\left\lfloor\alpha \frac{X}{\omega}\right\rfloor=X \div \beta$, we can use the decomposition $X=\widetilde{X} \beta+\widehat{X}$ for any integer $X \in \mathcal{H R}_{\omega}$ so $\widetilde{X}=X \div \beta \in \mathcal{H} \mathcal{R}_{\alpha}$ and $\widehat{X}=X \bmod \beta \in\{0, \ldots, \beta-1\}$. The integer $\tilde{X} \in \mathcal{H} \mathcal{R}_{\alpha}$ is interpreted as the result of the rescaling on $X$.

With this notations, we can rewrite the scheme (3) and we get the arithmetization of the Euler scheme (2) at the drawing scale $\alpha$ by:

$$
\left\{\begin{aligned}
\left(\widetilde{X}_{0}, \widetilde{Y}_{0}\right) & =(0,\lfloor\omega R\rfloor \div \beta) \\
\left(\widehat{X}_{0}, \widehat{Y}_{0}\right) & =(0,\lfloor\omega R\rfloor \bmod \beta) \\
\left(F_{n}^{1}, F_{n}^{2}\right) & =\left(\left(-\widetilde{Y}_{n} \beta-\widehat{Y}_{n}\right) \div \beta,\left(\widetilde{X}_{n} \beta+\widehat{X}_{n}\right) \div \beta\right) \\
\left(\widetilde{X}_{n+1}, \widetilde{Y}_{n+1}\right) & =\left(\widetilde{X}_{n}+\left(\widehat{X}_{n}+F_{n}^{1}\right) \div \beta, \widetilde{Y}_{n}+\left(\widehat{Y}_{n}+F_{n}^{2}\right) \div \beta\right) \\
\left(\widehat{X}_{n+1}, \widehat{Y}_{n+1}\right) & =\left(\left(\widehat{X}_{n}+F_{n}^{1}\right) \bmod \beta,\left(\widehat{Y}_{n}+F_{n}^{2}\right) \bmod \beta\right)
\end{aligned}\right.
$$

where the relevant variables are $\widetilde{X}_{i}$ and $\widetilde{Y}_{i}$.

The points $\left(\widetilde{X}_{i}, \widetilde{Y}_{i}\right)$ define a discrete circle which depends on the numbers $R$, $\alpha$ and $\beta$ denoted by $C_{d}^{\alpha}(0, R)$. Symmetrically, we can also consider the arithmetization of the Euler scheme (2) at the drawing scale $\beta$, which is the analogue of scheme (4), and is given by:

$$
\left\{\begin{aligned}
\left(\widetilde{X}_{0}, \widetilde{Y}_{0}\right) & =(0,\lfloor\omega R\rfloor \div \alpha) \\
\left(\widehat{X}_{0}, \widehat{Y}_{0}\right) & =(0,\lfloor\omega R\rfloor \bmod \alpha) \\
\left(F_{n}^{1}, F_{n}^{2}\right) & =\left(\left(-\widetilde{Y}_{n} \alpha-\widehat{Y}_{n}\right) \div \beta,\left(\widetilde{X}_{n} \alpha+\widehat{X}_{n}\right) \div \beta\right) \\
\left(\widetilde{X}_{n+1}, \widetilde{Y}_{n+1}\right) & =\left(\widetilde{X}_{n}+\left(\widehat{X}_{n}+F_{n}^{1}\right) \div \alpha, \widetilde{Y}_{n}+\left(\widehat{Y}_{n}+F_{n}^{2}\right) \div \alpha\right) \\
\left(\widehat{X}_{n+1}, \widehat{Y}_{n+1}\right) & =\left(\left(\widehat{X}_{n}+F_{n}^{1}\right) \bmod \alpha,\left(\widehat{Y}_{n}+F_{n}^{2}\right) \bmod \alpha\right)
\end{aligned}\right.
$$

where the relevant variables are $\widetilde{X}_{i}$ and $\tilde{Y}_{i}$. We get another discrete circle denoted by $C_{d}^{\beta}(0, R)$. In every case, the numerical experimentations show that connectedness of the digital curves $C_{d}^{\alpha}(0, R)$ and $C_{d}^{\beta}(0, R)$ is directly linked to the relative values of $\alpha$ and $\beta$. In the following section, we will establish a relation between these scale parameters in order to obtain 8-connectivity.

Remark. The schemes (4) and (5) describe two algorithms that are defined for every integer values of $\alpha$ and $\beta$ and not only for nonstandard values. Thus, despite the nonstandard framework used in the theoretical introduction of the arithmetization method, we finally get two standard families of discrete curves $C_{d}^{\alpha}(0, R)$ and $C_{d}^{\beta}(0, R)$ depending on two integer parameters $\alpha$ and $\beta$. The link with the nonstandard foundation is that, in some natural meaning, the discrete curves $C_{d}^{\alpha}(0, R)$ and $C_{d}^{\beta}(0, R)$ converge toward the continuous circle $C(0, R)$ when $\alpha$ and $\beta$ become infinitely large. 


\section{The Connectedness of $C_{d}^{\alpha}(0, R)$ and $C_{d}^{\beta}(0, R)$ Circle Arcs}

Graphical results (figures 1 and 2) show that the connectedness of the preceding discrete circles depends on the relative values of the drawing scales $\alpha$ and $\beta$. The section aim is to provide a condition on the scales that assure 8-connectivity for the discrete circle arcs of $C_{d}^{\alpha}(0, R)$ and $C_{d}^{\beta}(0, R)$. It must be noticed that these section results are independent of the nonstandard framework.

Let us consider the solution $\left(\left(\widetilde{X}_{n}, \widetilde{Y}_{n}\right)\right)_{0 \leq n}$ of the arithmetization of the Euler scheme (5) at the drawing scale $\alpha$ which is the discrete circle $C_{d}^{\alpha}(0, R)$. Let us call an arc of $C_{d}^{\alpha}(0, R)$ every subsequence of the form $\left(\left(\widetilde{X}_{n}, \widetilde{Y}_{n}\right)\right)_{k \leq n \leq k+p}$ for $k, p \in \mathbb{N}$ such that $p \geq 1$. In order to state the theorem that follows, let us introduce the square in $\mathbb{Z}^{2}$ centered at 0 with side length $2 \beta$ :

$$
R_{\beta}=\left\{(X, Y) \in \mathbb{Z}^{2} ;-\beta \leq X<\beta \text { and }-\beta \leq Y<\beta\right\} .
$$

Theorem 1. Every arc of $C_{d}^{\alpha}(0, R)$ in the square $R_{\beta}$ is 8-connected.

Proof. Let $\Gamma=\left(\left(\widetilde{X}_{n}, \widetilde{Y}_{n}\right)\right)_{k \leq n \leq k+p}$ be an arc of $C_{d}^{\alpha}(0, R)$ such that $\left(\widetilde{X}_{n}, \widetilde{Y}_{n}\right) \in$ $R_{\beta}$ for each $n=k, \ldots, k+p$. Then, $\Gamma$ is 8 -connected if and only if, for each $n=k, \ldots, k+p-1$, we have

$$
-1 \leq \widetilde{X}_{n+1}-\widetilde{X}_{n} \leq 1 \text { and }-1 \leq \widetilde{Y}_{n+1}-\widetilde{Y}_{n} \leq 1
$$

The proof is in two parts: in part (a) we will give a necessary and sufficient condition for the connectedness of $\Gamma$, in part (b) we will show that the condition $\Gamma \subset R_{\beta}$ is sufficient.
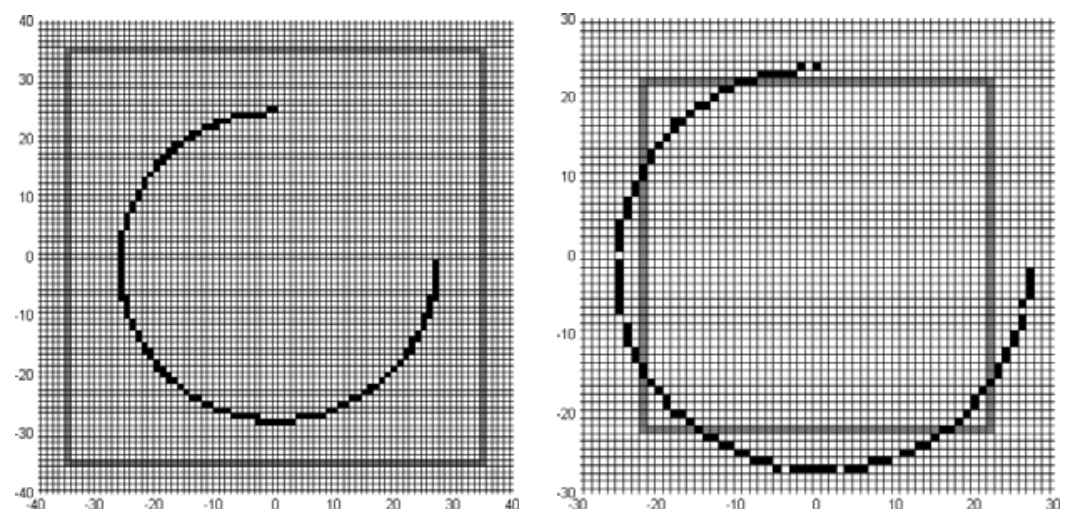

Fig. 1. Two examples of circle arcs at $\alpha$ scale (a): the one connected with $(R, \alpha, \beta)=$ $(1,25,35)$ (b) the other one disconnected with $(R, \alpha, \beta)=(1,24,22)$. The gray square represents the region $R_{\beta}$. 

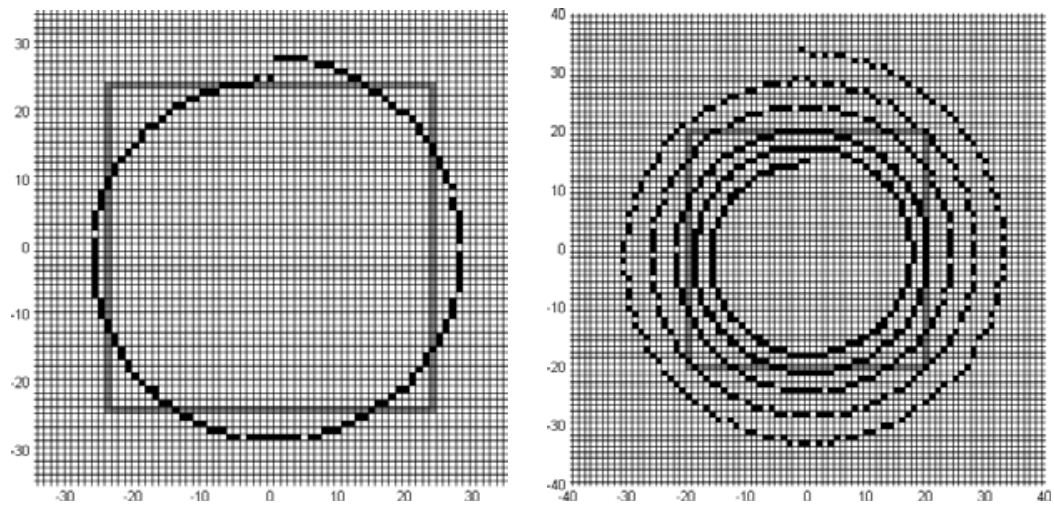

Fig. 2. The circle arcs of length (a) $\lfloor 2 \pi \beta\rfloor$ with $(R, \alpha, \beta)=(1,25,40)$ (b) $\lfloor 10 \pi \beta\rfloor$ with $(R, \alpha, \beta)=(1,15,20)$. The gray square represents the region $R_{\beta}$.

(a) Equivalent conditions: using the two schemes (3) and (4) and properties of the Euclidean division, we can see that the following conditions are equivalent:

$$
\begin{gathered}
-1 \leq \widetilde{X}_{n+1}-\widetilde{X}_{n} \leq 1 \text { and }-1 \leq \widetilde{Y}_{n+1}-\widetilde{Y}_{n} \leq 1 \\
-1 \leq\left(\widehat{X}_{n}+F_{n}^{1}\right) \div \beta \leq 1 \text { and }-1 \leq\left(\widehat{Y}_{n}+F_{n}^{2}\right) \div \beta \leq 1 \\
-\beta \leq \widehat{X}_{n}+F_{n}^{1}<2 \beta \text { and }-\beta \leq \widehat{Y}_{n}+F_{n}^{2}<2 \beta \\
-\beta-\widehat{X}_{n} \leq\left(-\widetilde{Y}_{n} \beta-\widehat{Y}_{n}\right) \div \beta<2 \beta-\widehat{X}_{n} \text { and }-\beta-\widehat{Y}_{n} \leq\left(\widetilde{X}_{n} \beta+\widehat{X}_{n}\right) \div \beta<2 \beta-\widehat{Y}_{n} \\
-\beta^{2}-\widehat{X}_{n} \beta \leq-\widetilde{Y}_{n} \beta-\widehat{Y}_{n}<2 \beta^{2}-\widehat{X}_{n} \beta \text { and }-\beta^{2}-\widehat{Y}_{n} \beta \leq \widetilde{X}_{n} \beta+\widehat{X}_{n}<2 \beta^{2}-\widehat{Y}_{n} \beta \\
-\beta^{2}-\widehat{X}_{n} \beta \leq-Y_{n}<2 \beta^{2}-\widehat{X}_{n} \beta \text { and }-\beta^{2}-\widehat{Y}_{n} \beta \leq X_{n}<2 \beta^{2}-\widehat{Y}_{n} \beta .
\end{gathered}
$$

Thus, $\Gamma$ is 8 -connected if and only if, for each $n=k, \ldots, k+p-1$, we have:

$$
-2 \beta^{2}+\widehat{X}_{n} \beta<Y_{n} \leq \beta^{2}+\widehat{X}_{n} \beta \text { and }-\beta^{2}-\widehat{Y}_{n} \beta \leq X_{n}<2 \beta^{2}-\widehat{Y}_{n} \beta \text {. }
$$

(b) Sufficient condition: Since $0 \leq \widehat{X}_{n} \leq \beta-1$ and $0 \leq \widehat{Y}_{n} \leq \beta-1$, we get the following two sequences of inequalities:

$$
\begin{gathered}
-2 \beta^{2}+\widehat{X}_{n} \beta \leq-\beta^{2}-\beta<-\beta^{2}<\beta^{2} \leq \beta^{2}+\widehat{X}_{n} \beta \\
-\beta^{2}-\widehat{Y}_{n} \beta \leq-\beta^{2}<\beta^{2}<\beta^{2}+\beta \leq 2 \beta^{2}-\widehat{Y}_{n} \beta .
\end{gathered}
$$

Thus, if $-\beta^{2} \leq X_{n}<\beta^{2}$ and $-\beta^{2} \leq Y_{n}<\beta^{2}$, then the condition (77) is verified. It is easy to see that, for $a \in \mathbb{Z}$ and $b \in \mathbb{N} \backslash\{0\}$, we have:

$$
-b^{2} \leq a<b^{2} \Longleftrightarrow-b \leq a \div b<b .
$$

Since $\tilde{X}_{n}=X_{n} \div \beta$ and $\tilde{Y}_{n}=Y_{n} \div \beta$, the condition $-\beta^{2} \leq X_{n}<\beta^{2}$ is equivalent to $-\beta \leq \widetilde{X}_{n}<\beta$ and $-\beta^{2} \leq Y_{n}<\beta^{2}$ to $-\beta \leq \widetilde{Y}_{n}<\beta$. Hence, the 8-connectedness of $\Gamma$ is a consequence of the condition:

$\forall n=k, \ldots, k+p-1 \quad-\beta \leq \widetilde{X}_{n}<\beta$ and $-\beta \leq \widetilde{Y}_{n}<\beta$. 
Let us call an initial arc of $C_{d}^{\alpha}(0, R)$ every arc of the form $\left(\left(\widetilde{X}_{n}, \widetilde{Y}_{n}\right)\right)_{0 \leq n \leq p}$ for $p \geq 1$. That is to say, an arc starting from the initial value $\left(\widetilde{X}_{0}, \widetilde{Y}_{0}\right)$.

Corollary 1. If $\alpha R<\beta$, then $C_{d}^{\alpha}(0, R)$ has an initial arc which is 8-connected.

Proof. According to the preceding theorem, $C_{d}^{\alpha}(0, R)$ has an initial arc which is 8-connected if $-\beta \leq \widetilde{X}_{0}<\beta$ and $-\beta \leq \widetilde{Y}_{0}<\beta$. Since $\left(\widetilde{X}_{0}, \widetilde{Y}_{0}\right)=(0,\lfloor\omega R\rfloor \div \beta)$, this property is reduced to each of the following equivalent condition:

$$
-\beta \leq\lfloor\omega R\rfloor \div \beta<\beta \Leftrightarrow-\beta^{2} \leq\lfloor\omega R\rfloor<\beta^{2} \Leftrightarrow-\beta \leq \alpha R<\beta .
$$

Thus we get the result since $\alpha R \geq 0$.

In the same way, we can consider the arithmetization of the Euler scheme at the drawing scale $\beta$. In this case, we have the following result:

\section{Theorem 2.}

- Every arc of $C_{d}^{\beta}(0, R)$ in the square $R_{\beta}$ is 8-connected.

- If $R<1$, then $C_{d}^{\beta}(0, R)$ has an initial arc which is 8-connected.

Proof. The proof is similar to the previous one.

Figures 1 and 2 provide a graphic illustration of these properties. Only graphic illustrations at drawing scale $\alpha$ are proposed here. The first one represents a discrete connected circle arc with $R=1,(\alpha, \beta)=(25,35)$ and a disconnected one with $R=1,(\alpha, \beta)=(24,22)$. The region $R_{\beta}$ is also displayed (in gray). The second represents the discrete connected circle arc with length $\lfloor 2 \pi \beta\rfloor$ with $R=1,(\alpha, \beta)=(25,40)$ and the initial arc of $C_{d}^{\alpha}(0, R)$ with length $\lfloor 10 \pi \beta\rfloor$ with $R=1,(\alpha, \beta)=(25,30)$ which provides a spiral. This last one brings to light discontinuity outside of the square (each arc in the square is 8-connected while the ones which are outside can be disconnected).

If the arc is composed of $N=\lfloor 2 \pi \beta\rfloor$ discrete points we should obtain a full circle but, in general, its first point is not connected to its last point (cf figure 2(a)). This arc is not a very good discretization of the underlying circle. The reason is that the Euler's scheme, as it is well known, accrues a small error at each integration step. When the curve is run one time the error is sufficiently large to "miss" the first point of the arc. This phenomenon depends of course on the integration scheme we use and also on the translation of the arithmetized scheme into the real domain. This is studied in the next section.

\section{Global Error Estimate}

The aim of this section is to study the error of our arithmetization method. To do that, let us describe a more general framework in which our result will be placed. Let $f: U \rightarrow \mathbb{R}$ be a function of class $C^{1}$ defined on an open set $U$ of $\mathbb{R} \times \mathbb{R}^{n}$. We suppose that $f$ is standard (that is to say independent of the 
nonstandard framework) and that $f$ and its partial derivatives are bounded in $U$. Let us consider the continuous Cauchy problem:

$$
\left\{\begin{array}{l}
z(a)=b \\
z^{\prime}(t)=f(t, z(t))
\end{array}\right.
$$

with $a \in \mathbb{R}, b \in \mathbb{R}^{n}, a$ and $b$ limited. We consider the solution $z:[a, c] \rightarrow \mathbb{R}^{n}$ of (8) where $c \in \mathbb{R}$ is limited and such that $a \not c$ (i.e. $a$ not infinitely close to c). This solution can be approximated by the Euler scheme:

$$
\begin{cases}\left(t_{0}, z_{0}\right) & =(a, b) \\ \left(t_{n+1}, z_{n+1}\right) & =\left(t_{n}+\frac{1}{\beta}, z_{n}+\frac{1}{\beta} f\left(t_{n}, z_{n}\right)\right)\end{cases}
$$

in which we choose an integration step $h=\frac{1}{\beta}$. For a infinitely large $\beta$ (i.e. $\beta \simeq+\infty$ ), we have $z_{n}$ infinitely close to $z\left(t_{n}\right)$ (i.e. $z_{n} \simeq z\left(t_{n}\right)$ ) for $0 \leq n \leq N$ with $N=\lfloor\beta c\rfloor$. Our arithmetization method allows us to introduce a similar scheme working only with integer variables:

$$
\left\{\begin{array}{l}
\left(T_{0}, Z_{0}\right)=(\lfloor\omega a\rfloor,\lfloor\omega b\rfloor) \\
\left(T_{n+1}, Z_{n+1}\right)=\left(T_{n}+\alpha, Z_{n}+F\left(T_{n}, Z_{n}\right) \div \beta\right)
\end{array}\right.
$$

where $F\left(T_{n}, Z_{n}\right)=\left\lfloor\omega f\left(\frac{T_{n}}{\omega}, \frac{Z_{n}}{\omega}\right)\right\rfloor$. This scheme results from the mapping $\psi_{\omega}$ and by substitution of the division by $\beta$ by the Euclidean division by $\beta$.

Now using the reverse mapping $\varphi_{\omega}$, we define a new scheme working with real variables:

$$
\left\{\begin{array}{l}
\left(t_{0}^{\prime}, z_{0}^{\prime}\right)=\left(\frac{1}{\omega}\lfloor\omega a\rfloor, \frac{1}{\omega}\lfloor\omega b\rfloor\right) \\
\left(t_{n+1}^{\prime}, z_{n+1}^{\prime}\right)=\left(t_{n}^{\prime}+\frac{1}{\beta}, z_{n}^{\prime}+\frac{1}{\beta} g\left(t_{n}^{\prime}, z_{n}^{\prime}\right)\right)
\end{array}\right.
$$

where $t_{n}^{\prime}=\frac{T_{n}}{\omega}, z_{n}^{\prime}=\frac{Z_{n}}{\omega}$ and $g\left(t_{n}^{\prime}, z_{n}^{\prime}\right)=\frac{1}{\alpha}\left\lfloor\frac{1}{\beta}\left\lfloor\omega f\left(t_{n}^{\prime}, z_{n}^{\prime}\right)\right\rfloor\right\rfloor$. We call this scheme the trace of the arithmetized scheme (10). We can remark that the two schemes (9) and (11) are very similar.

Our purpose is to estimate the distance between the value $z_{n}^{\prime}$ of the trace of the arithmetized scheme and the value $z\left(t_{n}\right)$ of the solution of the Cauchy problem (8). This distance defines the global error of our arithmetization method.

In order to fix our distance notion, we choose the infinity norm $(\|x\|=$ $\left.\left\|\left(x_{1}, \ldots, x_{n}\right)\right\|=\max _{1 \leq i \leq n}\left|x_{i}\right|\right)$, and then we have the following result:

Theorem 3. There exists limited constants $\mathcal{L}, \mathcal{L}^{\prime}, \mathcal{L}^{\prime \prime} \in \mathbb{R}$ such that

$$
\forall n \in[[0, N]],\left\|z_{n}^{\prime}-z\left(t_{n}\right)\right\| \leq \frac{\mathcal{L}}{\omega}+\frac{\mathcal{L}^{\prime}}{\alpha}+\frac{\mathcal{L}^{\prime \prime}}{\beta} .
$$

Proof. In order to compute an upper-bound of $\left\|z_{n}^{\prime}-z\left(t_{n}\right)\right\|$, we split this expression in two parts:

$$
\left\|z_{n}^{\prime}-z\left(t_{n}\right)\right\| \leq\left\|z_{n}^{\prime}-z_{n}\right\|+\left\|z_{n}-z\left(t_{n}\right)\right\|
$$

where $z_{n}$ is the value the solution of the scheme (9) and then we find an upper bound separately for each part. The proof is thus split in two steps. By 
convention, to simplify the presentation of the following inequalities, all the indices $n$ are in $[[0, N]]$.

The first step is to compute an upper bound on $\left\|z_{n}-z_{n}^{\prime}\right\|$. To do so, we consider $\left\|z_{n+1}-z_{n+1}^{\prime}\right\|$. Using the usual triangular inequality, the schemes (9) and (11), we obtain:

$\left\|z_{n+1}-z_{n+1}^{\prime}\right\| \leq\left\|z_{n}-z_{n}^{\prime}\right\|+\frac{1}{\beta}\left\|\left(f\left(t_{n}, z_{n}\right)-f\left(t_{n}^{\prime}, z_{n}^{\prime}\right)\right)\right\|+\frac{1}{\beta}\left\|\left(f\left(t_{n}^{\prime}, z_{n}^{\prime}\right)-g\left(t_{n}^{\prime}, z_{n}^{\prime}\right)\right)\right\|$.

In order to bound the expression $\left\|\left(f\left(t_{n}^{\prime}, z_{n}^{\prime}\right)-g\left(t_{n}^{\prime}, z_{n}^{\prime}\right)\right)\right\|$, we consider $g\left(t_{n}^{\prime}, z_{n}^{\prime}\right)=$ $\frac{1}{\alpha}\left\lfloor\frac{1}{\beta}\left\lfloor\omega f\left(t_{n}^{\prime}, z_{n}^{\prime}\right)\right\rfloor\right\rfloor$. Using the integer part properties, this can be rewritten as

$$
g\left(t_{n}^{\prime}, z_{n}^{\prime}\right)=f\left(t_{n}^{\prime}, z_{n}^{\prime}\right)-\frac{1}{\omega}\left\{\omega f\left(t_{n}^{\prime}, z_{n}^{\prime}\right)\right\}-\frac{1}{\alpha}\left\{\frac{1}{\beta}\left\lfloor\omega f\left(t_{n}^{\prime}, z_{n}^{\prime}\right)\right\rfloor\right\}
$$

where $0 \leq\left\{\omega f\left(t_{n}^{\prime}, z_{n}^{\prime}\right)\right\}<1$ and $0 \leq\left\{\frac{1}{\beta}\left\lfloor\omega f\left(t_{n}^{\prime}, z_{n}^{\prime}\right)\right\rfloor\right\}<1$. Hence, we obtain $\left\|g\left(t_{n}^{\prime}, z_{n}^{\prime}\right)-f\left(t_{n}^{\prime}, z_{n}^{\prime}\right)\right\| \leq \frac{2}{\alpha}$.

Now, let us consider the expression $\left\|\left(f\left(t_{n}, z_{n}\right)-f\left(t_{n}^{\prime}, z_{n}^{\prime}\right)\right)\right\|$. Because $f$ is a Lipschitz function (due to the class $C^{1}$ condition) we know that there exists $L_{1} \in \mathbb{R}$, with $L_{1}$ limited, such that:

$$
\left\|f\left(t_{n}, z_{n}\right)-f\left(t_{n}^{\prime}, z_{n}^{\prime}\right)\right\| \leq L_{1}\left(\left|t_{n}-t_{n}^{\prime}\right|+\left\|z_{n}-z_{n}^{\prime}\right\|\right) .
$$

In this last expression $\left|t_{n}-t_{n}^{\prime}\right|$ is such that $\forall n$,

$$
\left|t_{n}-t_{n}^{\prime}\right|=\left|t_{n-1}-\frac{1}{\beta}-t_{n-1}^{\prime}+\frac{1}{\beta}\right|=\left|t_{0}-t_{0}^{\prime}\right|=\left|a-\frac{\omega a}{\omega}+\frac{\{\omega a\}}{\omega}\right| \leq \frac{1}{\omega} .
$$

Combining all the previous inequalities we obtain:

$\left\|z_{n+1}-z_{n+1}^{\prime}\right\| \leq\left\|z_{n}-z_{n}^{\prime}\right\|\left(1+\frac{L_{1}}{\beta}\right)+\frac{L_{1}}{\beta \omega}+\frac{2}{\omega} \leq\left\|z_{n}-z_{n}^{\prime}\right\|\left(1+\frac{L_{1}}{\beta}\right)+\frac{L_{1}}{\omega}+\frac{2}{\omega}$.

This is transformed as

$$
\epsilon_{n+1} \leq \epsilon_{n}\left(1+\frac{L_{1}}{\beta}\right)+\frac{L_{2}}{\omega}
$$

where $L_{2}=2+L_{1}$ and $\epsilon_{n+1}=\left\|z_{n+1}-z_{n+1}^{\prime}\right\|$. The following well-known lemma is now used.

Lemma 1 (Arithmetic-Geometric Sequence Properties). Let $e_{n}$ be a sequence of $\mathbb{R}^{+}$. If $e_{n+1} \leq A e_{n}+B$ with $A, B \in \mathbb{R}^{+}$then

$$
\forall n, e_{n} \leq A^{n} e_{0}+B \frac{A^{n}-1}{A-1} .
$$

Thanks to this lemma, we express $\epsilon_{n}$ according to $\epsilon_{0}$ and obtain a bound for $\epsilon_{n}$ :

$$
\epsilon_{n} \leq \epsilon_{0}\left(1+\frac{L_{1}}{\beta}\right)^{n}+\frac{L_{2}}{\omega}\left(\frac{\left(1+\frac{L_{1}}{\beta}\right)^{n}-1}{\left(1+\frac{L_{1}}{\beta}\right)-1}\right) .
$$


Since $\frac{1}{\omega}$ is an upper-bound for $\epsilon_{0}=\left\|z_{0}-z_{0}^{\prime}\right\|$, we obtain:

$$
\epsilon_{n} \leq \frac{1}{\omega}\left(1+\frac{L_{1}}{\beta}\right)^{n}+\frac{L_{2}}{L_{1}} \frac{1}{\alpha}\left(\left(1+\frac{L_{1}}{\beta}\right)^{n}-1\right) .
$$

In order to find an upper bound of $\left(1+\frac{L_{1}}{\beta}\right)^{n}$, let us consider this other wellknown lemma:

Lemma 2. $(1+u)^{n} \leq e^{n u}, \forall n \in \mathbb{N}, \forall u \geq 0$

The inequality $\frac{n}{\beta} \leq(c-a)$ expresses that the iteration number $n$ is upperbounded by the product of the length of the integration interval (that is a limited number) by $\beta$. Moreover, the exponential of a limited number is a limited number. Hence, using lemma 2 , $\left(1+\frac{L_{1}}{\beta}\right)^{n}$ is bounded by a limited number. So, there exists limited constants $L_{3}, L_{4} \in \mathbb{R}$ such that:

$$
\left\|z_{n}-z_{n}^{\prime}\right\| \leq \frac{1}{\omega} L_{3}+\frac{1}{\alpha} L_{4} .
$$

The second step consists in computing an upper bound for $\left\|z_{n}-z\left(t_{n}\right)\right\|$ of equation (13). To do so, we consider $\left\|z_{n+1}-z\left(t_{n+1}\right)\right\|$.

Let us use the Taylor expansion of $z\left(t_{n+1}\right)$, there exists $\left.\gamma_{n} \in\right] t_{n}, t_{n}+h[$ such that:

$$
z\left(t_{n+1}\right)=z\left(t_{n}+h\right)=z\left(t_{n}\right)+h z^{\prime}\left(t_{n}\right)+\frac{h^{2}}{2} z^{\prime \prime}\left(\gamma_{n}\right) .
$$

Since $] t_{n}, t_{n}+h\left[\subset[a, c], z^{\prime \prime}\left(\gamma_{n}\right) \leq\left\|z^{\prime \prime}\right\|_{[a, c]}\right.$ where $\left\|z^{\prime \prime}\right\|_{[a, c]}$ is the norm of $z^{\prime \prime}$ in the interval $[a, c]$. So from (18) we have:

$\left\|z_{n+1}-z\left(t_{n+1}\right)\right\| \leq\left\|z_{n}-z\left(t_{n}\right)\right\|+\frac{1}{\beta}\left\|f\left(t_{n}, z_{n}\right)-f\left(t_{n}, z\left(t_{n}\right)\right)\right\|+\frac{1}{2}\left(\frac{1}{\beta}\right)^{2}\left\|z^{\prime \prime}\right\|_{[a, c]}$.

As previously, we set $\epsilon_{n+1}^{\prime}=\left\|z_{n+1}-z\left(t_{n+1}\right)\right\|$ and we obtain the inequality:

$$
\epsilon_{n+1}^{\prime} \leq \epsilon_{n}\left(1+\frac{1}{\beta} L_{1}\right)+\frac{1}{2}\left(\frac{1}{\beta}\right)^{2} L_{5},
$$

where $L_{5} \in \mathbb{R}$ is limited and such that $\left\|z^{\prime \prime}\right\|_{[a, c]} \leq L_{5}$. Now we can use again lemmas 1 and 2 and we obtain an upper bound for $\left\|z_{n}-z\left(t_{n}\right)\right\|$. There exists a limited constant $L_{6} \in \mathbb{R}$ such that $\left\|z_{n}-z\left(t_{n}\right)\right\| \leq \frac{1}{\beta} L_{6}$. Hence, from this last inequality and inequality (17) we can conclude that there exists limited constants $\mathcal{L}, \mathcal{L}^{\prime}$ and $\mathcal{L}^{\prime \prime}$ in $\mathbb{R}$ such that: $\left\|z_{n}^{\prime}-z\left(t_{n}\right)\right\| \leq \frac{\mathcal{L}}{\omega}+\frac{\mathcal{L}^{\prime}}{\alpha}+\frac{\mathcal{L}^{\prime \prime}}{\beta}$.

In the particular case of the circles described in section 2 , the exact solution is $z(t)=R e^{i\left(t+\frac{\pi}{2}\right)}, t \in[0,2 \pi]$ and we obtain:

$$
\left\|z_{n}^{\prime}-z\left(t_{n}\right)\right\| \leq \frac{e^{2 \pi}}{\omega}+\frac{2\left(e^{2 \pi}-1\right)}{\alpha}+\frac{\frac{R\left(e^{2 \pi}-1\right)}{2}}{\beta} .
$$


In the field of numerical analysis, integration schemes [18] come with an approximation order. This gives an idea of the numerical quality of the computed solutions. Until now, the order of arithmetized schemes had never been studied and the previous theorem allows us to define an approximation order of an arithmetized integration scheme which is the equivalent of the classical approximation order used in numerical analysis.

The classical approximation order is defined as the biggest integer such that there exists a constant $\mathcal{C} \in \mathbb{R}$ verifying $\left\|z_{n}-z\left(t_{n}\right)\right\| \leq \mathcal{C} h^{p}$, where $h$ is the integration step (small by definition). Each integration scheme has a precise order. For example, Euler method is of order 1. Other methods, such as the Heun scheme [18], is of order 2. From theorem 3. we propose now a definition for the approximation order of the Euler arithmetized scheme (10):

Definition 1 (Order). The Euler arithmetized scheme (10) is of order $p$ if there exists a limited number $\mathcal{L} \in \mathbb{R}$ such that:

$$
\forall n \in[[0, N]],\left\|z_{n}^{\prime}-z\left(t_{n}\right)\right\| \leq \frac{\mathcal{L}}{\beta^{p}} .
$$

With this definition, the following corollary gives a sufficient condition to have order 1 for the arithmetized Euler scheme (10).

Corollary 2. If $\frac{\beta}{\alpha}$ is limited then the arithmetized scheme (10) is of order 1 .

Proof. There is a limited number $\lambda$ such that $\beta=\lambda \alpha$. Then, from theorem 3 $\left\|z_{n}^{\prime}-z\left(t_{n}\right)\right\| \leq \frac{1}{\beta}\left(\frac{\mathcal{L}}{\alpha}+\mathcal{L}^{\prime} \lambda+\mathcal{L}\right)$ and $\frac{\mathcal{L}}{\alpha}+\mathcal{L}^{\prime} \lambda+\mathcal{L}$ is clearly limited.

The order of arithmetized integration schemes is currently under investigation by the authors and the previous definition can be extended to other schemes such as the Heun [18] integration scheme. However, the status of this approximation order is not so clear. For example, using a theorem similar to theorem 3 . the arithmetized Heun scheme is of order at most 2 as expected from its corresponding original scheme. But, when studying connectedness, the condition found seems to impose that the obtained numerical solution is only of order 1 . Numerical experiments show nonetheless that obtained circle arcs are "better" than those obtained by the arithmetized Euler scheme. This is still largely an open question.

\section{Conclusion}

In this paper we studied the arithmetization of the Euler integration scheme over the differential equation defining a circle. This work reconsiders a previous work done by Holin [15] and studies carefully the arithmetization process.

New results have been proposed; connectedness criteria for the two families of discrete circular arcs, a global error estimate between the arithmetized scheme trace and the initial Euler scheme, and a first definition of the approximation order of an arithmetized integration scheme. These results provide new insights in the way a curve can be approximated by the arithmetization process. 
Our next goal is to generalize the preceding results to integration schemes with higher approximation orders. This will gives us a deeper understanding of the arithmetization process and improve the definition of the approximation order of an arithmetized integration scheme. Numerical experiments are under way. We have already observed that better circle arcs are obtained by Heun's method (as obtained by Holin) and that connectedness criteria still remain the same. This latter observation is surprising and is not yet fully understood.

\section{References}

1. Bresenham, J.: Algorithm for computer control of a digital plotter. ACM trans. Graphics 4, 25-30 (1965)

2. Bresenham, J.: A linear algorithm for incremental digital display of circular arcs. Comm. of ACM 20, 100-106 (1977)

3. Reveillès, J.P.: Mathématiques discrètes et analyse non standard. In: [19], pp. $382-390$

4. Reveillès, J.P.: Géométrie discrète, Calcul en nombres entiers et algorithmique. PhD thesis, Université Louis Pasteur, Strasbourg, France (1991)

5. Reveillès, J.P., Richard, D.: Back and forth between continuous and discrete for the working computer scientist. Annals of Mathematics and Artificial Intelligence, Mathematics and Informatic 16, 89-152 (1996)

6. Andres, E.: Discrete circles, rings and spheres. Computer and Graphics 18, 695-706 (1994)

7. Nelson, E.: Internal set theory: A new approach to nonstandard analysis. Bulletin of the American Mathematical Society 83, 1165-1198 (1977)

8. Robinson, A.: Non-standard analysis. American Elsevier, New York (1974)

9. Harthong, J.: Une théorie du continu. In: Barreau, H., Harthong, J. (eds.) La mathématique non standard, Editions du CNRS, Paris, pp. 307-329 (1989)

10. Diener, M.: Application du calcul de Harthong-Reeb aux routines graphiques. In: [19], pp. 424-435

11. Harthong, J., Reeb, G.: Intuitionnisme 84. In: Barreau, H., Harthong, J. (eds.) La mathématique nonstandard, CNRS, pp. 213-252 (1989)

12. Harthong, J.: Éléments pour une théorie du continu. Astérisque 109/110, 235-244 (1983)

13. Fuchs, L., Largeteau-Skapin, G., Wallet, G., Andres, E., Chollet, A.: A first look into a formal and constructive approach for discrete geometry using nonstandard analysis. In: Coeurjolly, D., Sivignon, I., Tougne, L., Dupont, F. (eds.) DGCI 2008. LNCS, vol. 4992, pp. 21-32. Springer, Heidelberg (2008)

14. Chollet, A., Wallet, G., Fuchs, L., Largeteau-Skapin, G., Andres, E.: Insight in discrete geometry and computational content of a discrete model of the continuum. Pattern recognition (2009)

15. Holin, H.: Harthong-Reeb analysis and digital circles. The Visual Computer 8, 8-17 (1991)

16. Holin, H.: Harthong-Reeb circles. Séminaire non standard, Univ. de Paris 7 2, 1-30 (1989)

17. Holin, H.: Some artefacts of integer computer circles. Ann. Math. Artif. Intell. 16, 153-181 (1996)

18. Quarteroni, A., Sacco, R., Saleri, F.: Numerical mathematics. Springer, Heidelberg (2000)

19. Salanskis, J.M., Sinaceurs, H. (eds.): Le Labyrinthe du Continu. Springer, Heidelberg (1992) 